\title{
CUTANEOUS SARCOIDOSIS
}

\author{
By Gordon B. Mitchell-Heggs, M.D., F.R.C.P. and \\ Michael Feiwel, M.B., Ch.B.; M.R.C.P. \\ Department of Dermatology, St. Mary's Hospital, W.2
}

Sarcoidosis of the skin is often a striking picture and led to its recognition as a disease entity. For the patient, its importance lies in disfigurement more than in disability. For the clinician, it may provide a ready means of diagnosis towards which one glance may give a clue. In addition, the skin has played an important rôle in the study of aetiology. The reactions to injected tuberculin, the response to B.C.G. inoculation, and to Kveim antigen are some of the ways in which the skin has been tested in sarcoidosis.

\section{Sarcoidosis}

The aetiology is not definitely established. The disorder involves the reticulo-endothelial system and many organs, including the lungs, the lymph nodes, the bones, the heart, the uveal tract, the skin and so on may be affected, although it is generally confined to a few situations. Now recognized more commonly, the onset is mainly between the tenth and fiftieth year. Sarcoidosis. may be heralded by prodromal symptoms of fatigue and malaise, dyspnoea and cough, anorexia, weight loss and fever, any one of which may be the dominant feature of the illness. Sometimes a serious symptom marks the onset. The course of the disease is then erratic, many relapses and remissions may occur, and it is difficult and sometimes impossible to judge its natural course and to assess its response to treatment.

The process of sarcoidosis is essentially benign, but may nevertheless bring about death by interference with the function of the heart, the brain, the liver or kidney, or through some other complication.

\section{Cutaneous Aspects of Sarcoidosis Diagnosis}

Skin manifestations will help in two ways. The clinician, finding a disease picture suggestive of sarcoidosis, will search for dermatological features and biopsy one to give the characteristic histology. Even where none will be found at the time, they may appear later. Or the patient may present skin lesions making sarcoidosis likely. After searching for systemic features, a skin biopsy is again an easy means of establishing the diagnosis.

In either case, the clinician is helped if he carries in his mind's eye the varying aspects of cutaneous sarcoidosis. At the same time, conditions resembling sarcoidosis of the skin must be differentiated. This is not easy because the eye needs practice and neither description nor photograph can adequately convey the subtleties of the makeup of a skin lesion on which a diagnosis rests.

\section{Clinical Manifestations}

The picture of the skin is a varied one and classification based on the early descriptions is into four types: Boeck's sarcoid, subcutaneous sarcoid of Darier-Roussy, lupus pernio of Besnier and erythrodermic sarcoid. Pautrier's monograph (I940) gives a full account.

Boeck's sarcoid: This is the most common type. Sharply defined papules or nodules may occur symmetrically; over face, shoulders and extensor surfaces of arms mainly, but may be found anywhere on the body, even on the mucous membranes. Ulceration is practically unknown.

(i) Papules. They usually erupt rather suddenly, and are well-defined, firm, pinkish-red or reddish-brown. They are smooth or scaly, sparsely distributed or in clusters, and slightly infiltrated. Their evolution may extend over several years with remissions and relapses, but finally the lesions flatten, leaving faint pigmented or erythematous areas.

(ii) Nodules. These are fewer in number but larger, and red, reddish-brown, or mauve in colour. They are smooth, firm, infiltrated and moveable over the underlying structures. Diascopy shows the resemblance of the small yellowgrey foci that make up the lesions, to the apple jelly nodules of lupus vulgaris. Early nodules look bright red, then as they grow larger, they become less vivid, and when fully evolved, they may remain static for years. Then, as the centre slowly flattens, the colour darkens leaving finally pigmented areas crossed by telangiectases. Whitish scarring with slight infiltration may also result. 


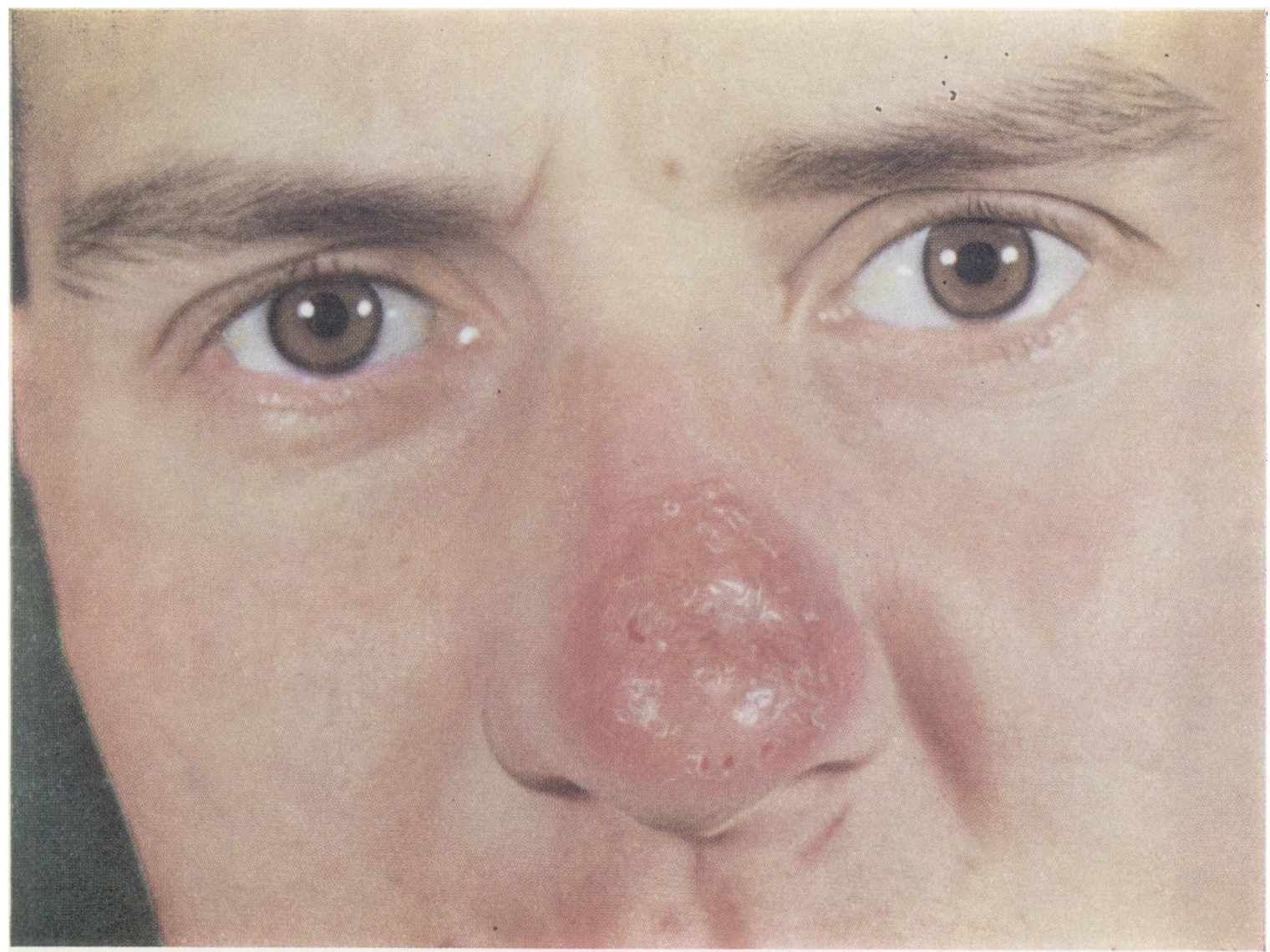

FIG. 1.-Boeck's sarcoid. Reddish-brown hue; infiltrated, clearly defined, smooth lesion, without telantiectasia.

(iii) Plaques. These show a tendency to central clearing, so that annular and circinate figures form, with telôngiectatic, raised edges.

Subcutaneous sarcoid, Darier and Roussy: Round or oval nodules are deeply seated, and may be skin coloured, blue or purplish. They vary in size, usually from $\mathrm{I} \mathrm{cm}$. to $4 \mathrm{~cm}$. in diameter. They develop slowly on the trunk and the legs (here resembling acrocyanosis) without subjective symptoms, and rarely exceed twenty in number. This type is not common and may occur with Boeck's sarcoid.

Lupus Pernio, Besnier. The smooth, shiny, purple nodules of lupus pernio can be distinguished from chilblain lupus (lupus erythematosus) by the absence of pain and their persistence during the summer. In lupus pernio, systemic sarcoidosis is frequently concurrent. The eruption is symmetrical, occurring mainly on the ears, cheeks, forehead, nose, dorsa of the hands, fingers and toes. The nose, most commonly affected, may have a disfiguring bulbous look. Fingers and toes may be the site of osteitis cystica, so that swellings result both from bone changes and cutaneous infiltration. The skin of lupus pernio is thick and indurated, with a network of telangiectases. Mutilating varieties have been reported.

Angio-lupoid, Brocq-Pautrier: A well-defined, indurated, solitary plaque or nodule, occurring on or around the nose, and affecting mainly middle-aged women, and round, cherry-sized and purplish in colour, with yellowish patches and obvious telangiectases. The yellow colour predominates under diascopy. Evolution is very slow, with little tendency to spontaneous regression.

Erythrodermic Sarcoid. An uncommon form (reviewed by Wigley and Musso, 1951). Sometimes associated with other types of cutaneous sarcoidosis, erythrodermic areas may be single, but are more often multiple. Found anywhere including the scalp, they are of differing shades of red and on diascopy show no yellow miliary lupoid foci but diffuse yellow staining. Of various shapes and sizes, they may enclose normal skin 
and have clear-cut or ill-defined edges. Infiltration is absent, slight or moderate. Scales, if present, are fine and transparent or coarse and adherent but readily removed. Often of rapid onset, the eruption lasts a few months to a few years or longer (even permanently), with remissions and relapses at times. When it subsides it leaves no trace, or else slight pigmentation.

Rare forms. These include a lichenoid type with a resemblance to lichen planus, an acute ulcerating type and a miliary form. Involvement of the scalp in sarcoidosis may lead to partial or complete alopecia.

Erythema Nodosum. One of the most interesting recent advances in our knowledge of sarcoidosis is that erythema nodosum may be one of its early manifestations. It emphasizes that sarcoidosis may, along with other processes, produce a nonspecific picture. When erythema nodosum is part of a syndrome and a negative Mantoux reaction and bilateral symmetrical hilar adenopathy accompany, sarcoidosis becomes the likely cause. Erythema nodosum is clinically distinctive. It appears to be an allergic reaction and antigens are of virus, bacterial, fungal or drug origin. (The unknown cause of sarcoidosis, possibly having its source of entry in the lungs to produce hilar gland enlargement, would be one of these antigens.)

Clinical Features. The swellings may be accompanied by fever and joint pains which settle as the swellings subside. The raised nodules arise mostly on the front of the shins, but also on the arms, trunk or face. They vary from about $\mathrm{r} \mathrm{cm}$. to about $5 \mathrm{~cm}$. or more in diameter, are pink or red at first, then become more livid showing the colour changes of a bruise and leaving a little residual staining. They are smooth, soft, roundish and tender on pressure. Additional crops can occur but the trouble is over in a few weeks or a month or two. There is no breaking down. It is probable that the initial change is vascular, for in the histology the larger veins in the upper part of the subcutaneous tissue show endothelial proliferation and the vessel wall infiltrated and surrounded by the inflammatory cells. Later foci of epithelioid cells appear with giant cells, usually of the foreign body type inside or outside these foci, and there is a resemblance to tuberculosis.

Scars, Pseudotuberculoma Silicoticum, Tattoos. The occurence of sarcoidosis in abnormal skin is not unusual and may be early. Infiltration of scars by sarcoid tissue may only produce a change of colour. They become reddish-brown or livid, with no palpable infiltration, or raised and even keloidal. A biopsy at this stage is typical of sarcoidosis. Scars containing particles of silica, engrained at the time of injury, may, many years later, become raised and firm with the formation

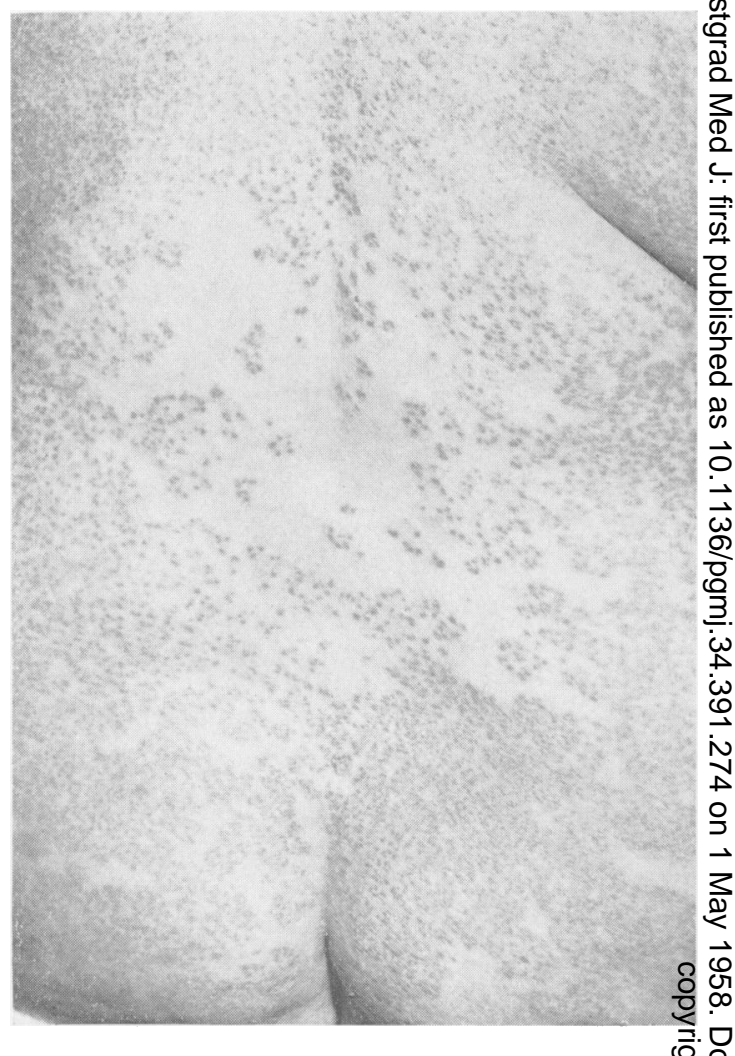

FIG. 2.-Sudden eruption of fine papular sarcolit, covering almost the whole skin surface within $14 \stackrel{\bar{O}}{-}$ days. Iritis and radiological lung changes present. (By courtesy of Miss $M$. Savory, photograph by Miss E. Mason, St. Fames' Hospital).

of a granuloma. When this is examined histo- $\frac{3}{3}$ logically, the picture of sarcoidosis may be seen. However, with the polarising microscope, doublyo refractile silica particles can be found within the sarcoid-like tissue. Two possibilities exist. Silica 3 may excite a tuberculoid- or sarcoid-like granulomad after a variable period, or the patient may have sarcoidosis localizing in a scar. In practice, botho occur. The granuloma may arise only where silica lies in the skin, but if the lesions are found? elsewhere, sarcoidosis becomes likely. Tattoos $>$ likewise may become raised and infiltrated. Again there are two possibilities. The patientu may have developed an allergy to the mercury? chromium or other constituent of the pigment, 0 possibly by further contact. Alternatively, thew tattoo has become the site of sarcoidosis (ot syphilis, leprosy, tuberculosis or even lupuso erythematosus).

\section{Histology}

While macroscopically pleomorphic, the microscopic appearance in the skin is remarkably uni- $\frac{\mathbb{N}}{\mathbb{D}}$ 


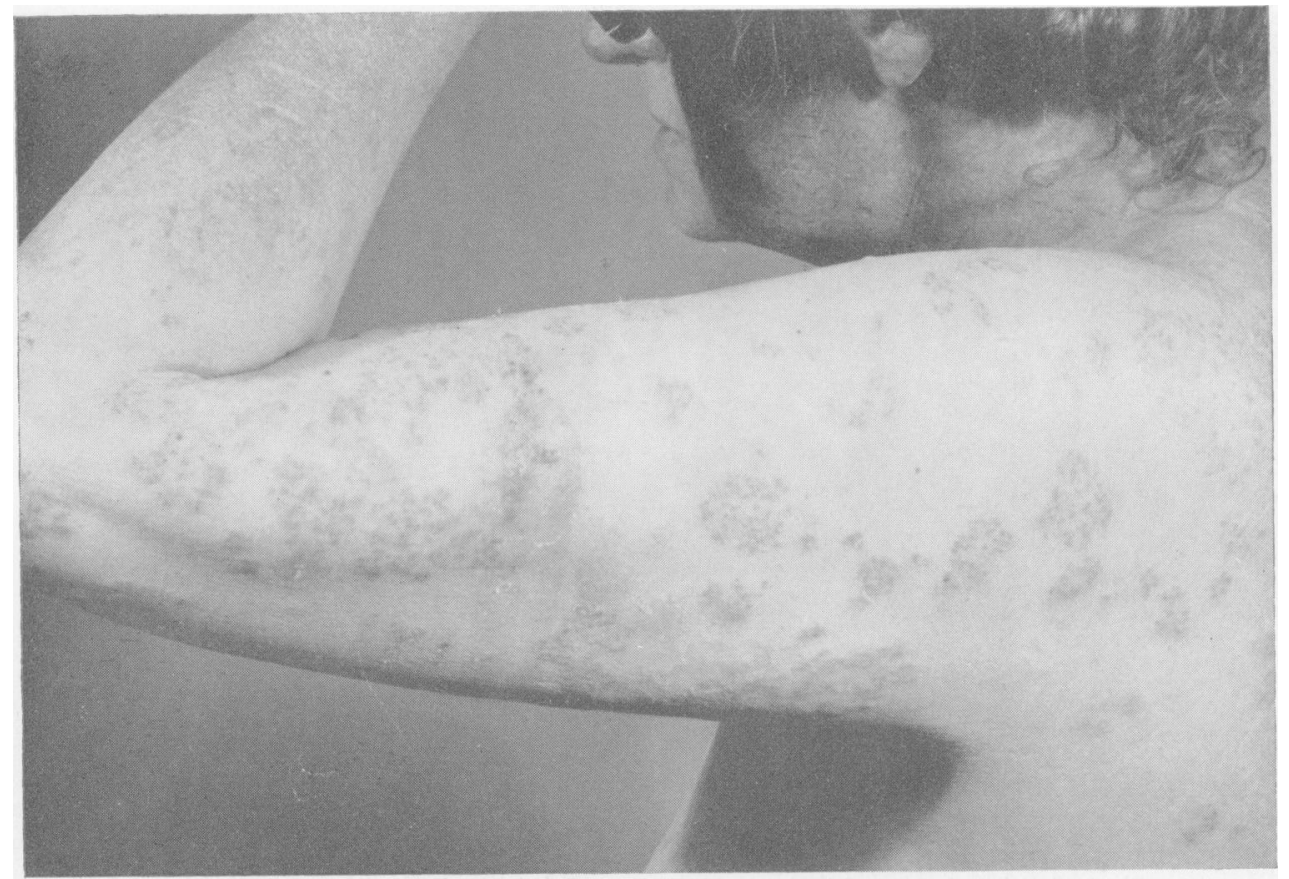

FIg. 3.-Papular sarcoid in clusters. Lung changes, enlargement of parotid glands, keratitis sicca.

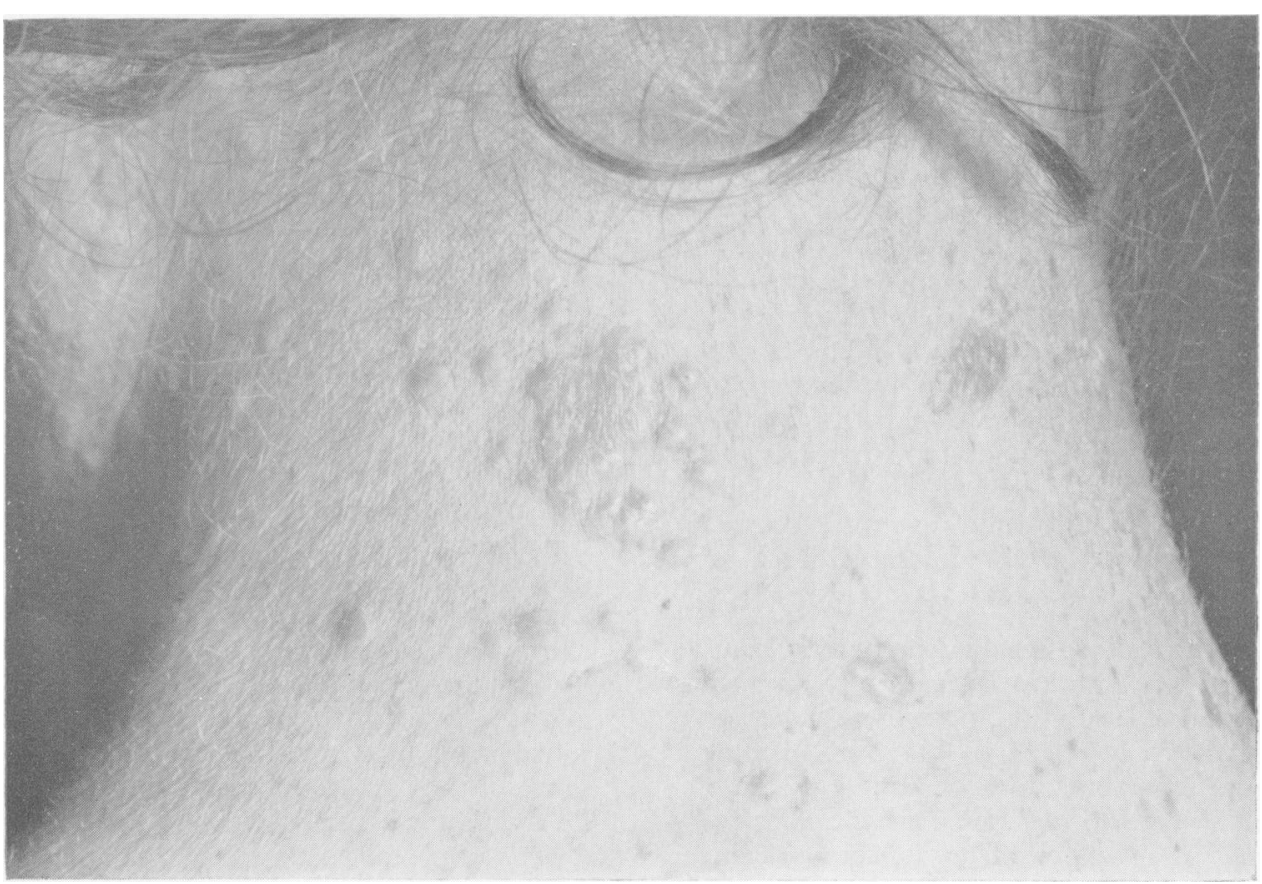

FIG. 4.-Nodular sarcoid in clusters. (Photographs I, 3 and 4 by Dr. P. Carderw, St. Mary's Hospital)

form, as in other organs. A granuloma of tuberculoid structure is composed mainly of circumscribed islands of epithelioid cells, and is situated in the dermis in Boeck's sarcoid, and in the subcutaneous tissue in Darier-Roussy sarcoid. Lupus pernio shows dilated capillaries in the upper part 


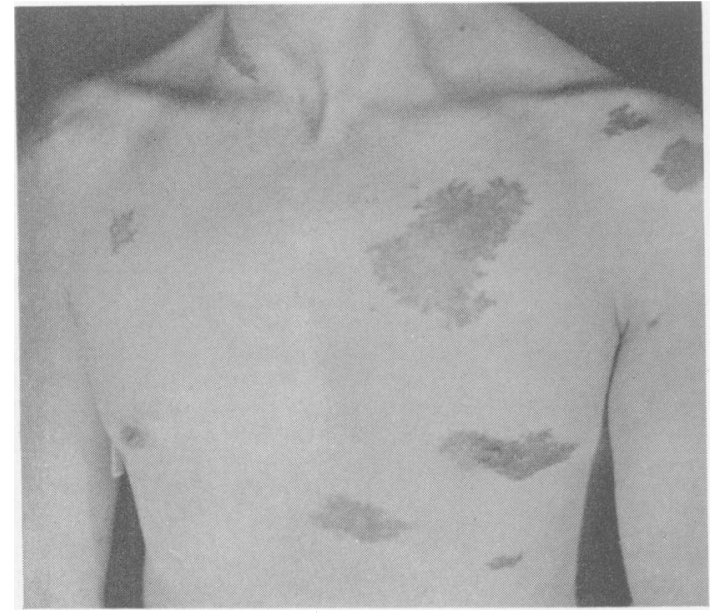

FIG. 5.-Irregular, discrete plaques of sarcoid over trunk.

of the dermis, and in erythrodermic sarcoid, the foci of epithelioid cells are small and histiocytes and lymphocytes surround superficial vessels. Lever (1954) details the histology in the skin as follows. In active lesions of sarcoidosis, the islands of epithelioid cells contain few Langhans giant cells or none at all. A slight and occasionally moderate admixture of lymphocytes is present, particularly at the margins of the epithelioid cell islands. Caseation necrosis is almost always absent. Rarely, some necrosis is found at the centre of the islands. A reticular network surrounds almost every epithelioid cell in most areas, but in others the fibres are concentrated at the periphery. In the healing phase, increasing fibrosis, usually starting at the periphery of the islands, obliterates the epithelioid cells and the lymphocytes. Reticular fibres transform into collagen fibres. A moderate number of giant cells, more of the foreign body type than Langhans cells, may occasionally contain so-called Schaumann inclusion bodies and asteroid inclusion bodies, but neither is specific for sarcoidosis.

\section{Differential Diagnosis}

The diagnosis of cutaneous sarcoidosis can be confirmed by systemic involvement, by an absent or weak tuberculin reaction, possibly by a positive Kveim test, as well as through its typical histology. Many conditions remain to be differentiated.

Those with Clinical and Histological Similarities: Lupus vulgaris. A patch of lupus, commonest on the face, often starts in childhood and extends gradually. Tuberculoid leprosy. Annular lesions resemble annular sarcoid. Finding thickened, palpable nerves, areas of anaesthesia, and, in the section, neural elements involved by tuberculoid infiltration and an occasional lepra bacillus help to

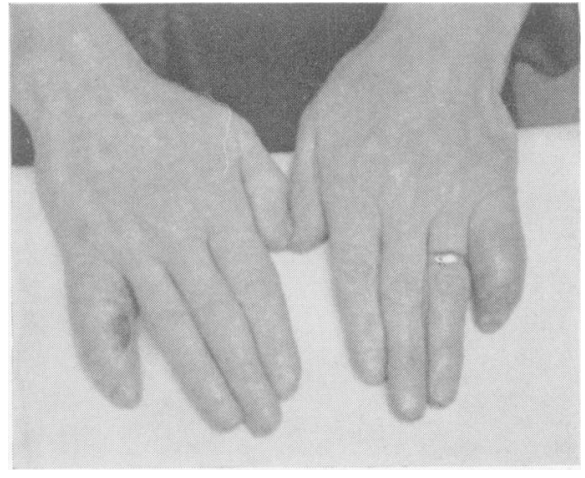

FI $_{\mathrm{G}}$. 6.-Lupus pernio, purple sausage-shaped fingers. (Photographs 5 and 6 by courtesy of Dr. F. Ingram.)

establish the diagnosis. The lepromin test is ususally positive in tuberculoid leprosy. Zirconium granuloma. Silicon granuloma has already been discussed in this article and that caused by berylliosis in this symposium (p. ). A sarcoidlike eruption in the axilla following the use of anti-perspirant sticks containing zirconium has been described (Rubin et al., 1956; Saunders, I957; Shelley, 1957).

Clinical Similarities, Histological Differences: Syphilis. A nodular syphilide is serpiginous as 8 rule. Gummatous ulceration and scarring tend te occur. On section, the granulation tissue showe many plasma cells, vascular changes are conspicu? ous and endarteritis is found. Acne agminata (Lupus miliaris disseminatum faciei). Small elevated papules on the face, especially the eyelids, show central caseation surrounded by tubercles under the microscope. Lupus erythematosus. Found on the nose, cheeks, ears, backs of fingers and the hands, one variety of lupus erythematosus (chilblain lupus of Hutchinson) may resemble lupus pernio. The changes in the epidermis, however, of follicular plugging and scaling, are reflected in the histology. Granuloma annulare. Small, firm, skin- or red-coloured papules or nodules arranged in rings are found and resemble a papular sarcoid. The characteristic histology shows an area of degenerated collagen surrounded by a ring of histiocytes, fibroblasts and lymphocytes. Erythema elevatum diutinum, with red to purple nodules, especially about the joints on the dorsa of the hands, the wrists and the elbows, may be a variant of granuloma annulare. The reticuloses (lymphomas). Red or purple papules or nodules are not infrequent, whether the disorder is leukaemic or not. Microscopically, a dense cellular infiltrate of the predominating cell type fills the dermis and extends deeply. Lymphocytoma cutis, the facial eosinophilic granuloma, Kaposi's idiopathic haemorrhagic sarcoma, xanthoma dis- 
seminatum, and urticaria pigmentosa. Conditions, related to the reticuloses which might occasionally give rise to difficulty. Necrobiosis lipoidica (diabeticorum vel non-diabeticorum), and granuloma disciformis chronica et progressiva. Sharply demarcated glazed patches, depressed and yellowish in the centre and violaceous at the periphery, usually found on the legs, are like an annular sarcoid. On section, necrobiotic areas of collagen surrounded by inflammatory cells are seen.

Clinical Differences, Histological Similarities: Cutaneous relapsing leishmaniasis. Although the miscroscope shows a tuberculoid picture and no Leishman-Donovan bodies, the history and the localization on exposed parts, usually the face, should establish the diagnosis. Rosacea with chronic lymphoedema (rosacea acuminatum), rosaceous tuberculide (Lewandowsky), and cheilitis granulomatosa and the Melkersson-Rosental triad (relapsing facial palsy, thick lips, and lingua plicata). It is only the histology that suggests sarcoid.

Other Causes of Erythema Nodosum. Tuberculosis, streptococcal infections, drugs such as sulphathiazole, as well as sarcoidosis, have been mentioned (this symposium, p. 24I). With increasing travel of all peoples, further causes of erythema nodosum require mention. Lymphogranuloma inguinale. A virus disease which begins as a small ulcer in the genital or perineal region and is followed by enlargement, sometimes painful, of the regional lymphatic glands. Fever and erythema nodosum may accompany. Leprosy. Erythema nodosum is one form of lepra reaction. It occurs during treatment by sulphones when lepra bacilli are multiplying and at the same time, are being rapidly destroyed by the drug. Deep fungus infection. In coccidiomycosis, cutaneous manifestations are usually late (coccidial granuloma). Erythema nodosum may occur in an early stage of the disease (San Joaquin Valley disease). Superficial fungus infections. When these are pustular, generalized allergic reactions - are not uncommon (trichophytids). Rarely a trichophytid is an erythema nodosum, especially in cases of pustular ringworm of the scalp (kerion).

Conditions Resembling Erythema Nodosum. If correctly diagnosed, they will exclude sarcoidosis. Erythema multiforme, toxic erythema. The eruption is more widespread and more profuse, and mucous membranes may be involved. Macules, papules or bullae make up the picture rather than nodules. Variations in colour (iris or target lesions) may be seen. Viruses, bacteria, fungi and drugs may produce it, but not tuberculosis or sarcoidosis, and it may also occur without obvious cause (and can be recurrent). Erythema induratum (Bazin's disease). Chronic purple nodules and

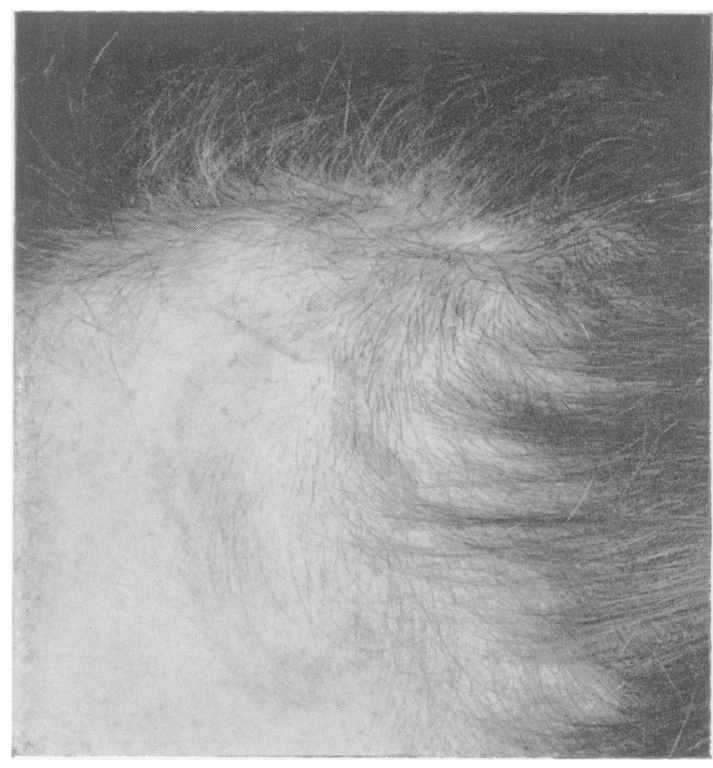

FIG. 7.--Annular sarcoid over left temple, confirmed by histology. (Photograph by Mr. R. Lunnon. By courtesy of Dr. R. M. B. MacKenna and Dr. F. R. Bettley, Dean of the Institute of Dermatology.)

plaques are deeply situated and ulceration is common. More on the backs of the calves and lower third of the legs than on the upper part of the shins, they occur usually in young women who have fat, cold legs and who may have had active tuberculosis in the past, especially glandular tuberculosis. Erythema induratum (Whitfield's type), nodular vasculitis. This is a non-tuberculous condition, more chronic than erythema nodosum. Females in an older age group than those with Bazin's disease have painful nodules, usually on the legs. The cause is not known, and it is uncommon. Erythema perino and erythrocyanosis. Young females with chilblain circulation may suffer from nodular vascular inflammatory changes at the backs of the calves, which may persist in the warmer weather. This form of chilblain, sometimes occurring in the absence of toe and finger involvement, may also affect the cold leg of past poliomyelitis. Chronic meningococcal septicaemia. In the epidemic of cerebrospinal fever during World War II, cases of chronic septicaemia were noted. There was fever, headache, pains in the joints and muscles, and an eruption of tender nodules, though perhaps more multiform and profuse than in erythema nodosum, sometimes with purpura. Sporadic cases continue. Thrombophlebitis. Fever and malaise accompany the painful nodules which are felt rather than seen along the course of the veins. Distribution tends, therefore, to be correspondingly linear. Nodules in 


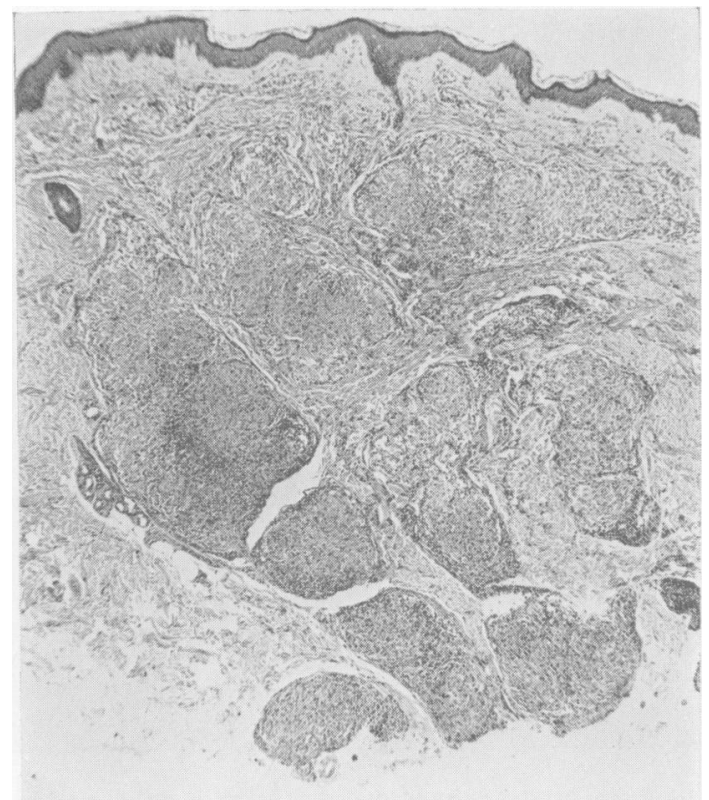

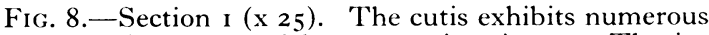
nodules separated by connective tissues. The individual nodules are composed of closely-set epithelioid cells and only a few round cells. The surrounding collagen looks normal.

lupus erythematosus and polyarteritis nodosa. Subacute systemic lupus erythematosus may show a nodular eruption on the legs owing to inflammatory involvement of the superficial vasculature. Without a typical rash and with a negative L.E. cell phenomenon, the diagnosis may be difficult, but the prolonged course and the polysymptomatic nature will arouse suspicion. In polyarteritis nodosa, the nodules are usually deeply situated and with overlying skin of normal colour. Macules, papules, petechiae, ulcers or livido racemosa may accompany the nodules. Nodular allergide of Gougerot (Maladie trisymptomatique). This has featured in the dermatological literature recently. There are purple nodules on the legs, but also macules, papules, purpura and ulcers. The condition is chronic and some cases end fatally. It may well be one presentation of the better recognized anaphylactoid purpura, polyarteritis. nodosa or lupus erythematosus. In some cases, cryoglobulins have been found. Relapsing febrile nodular nonsuppurative panniculitis (WeberChristian disease). Nodules and plaques appear in crops in the subcutaneous fat without a particular predilection for the lower limbs. Nodules are hard, and overlying skin is normal but red.

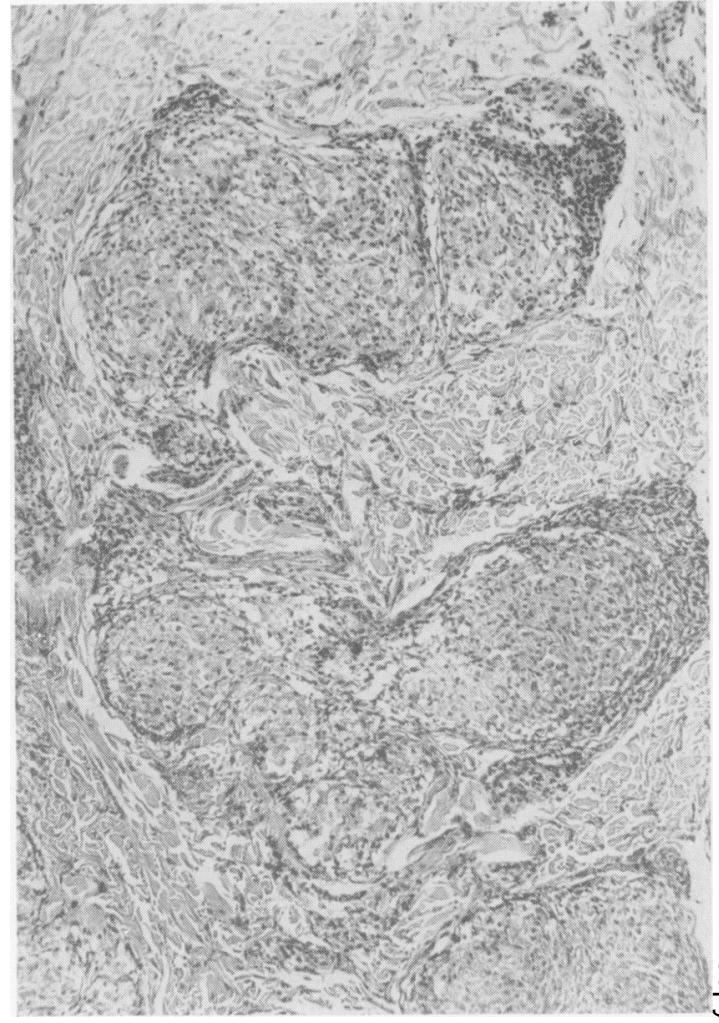

Fig. 9.-Section 2 (x 90). Detail of Section I. (Sections I and 2 by courtesy of Dr. H. Haber, Institute of Dermatology.)

Later the lesions fibrose and the skin becomes depressed, or the mass may liquefy and discharge.

\section{Treatment}

A decision on the use of steroids will depend on the overall picture of sarcoidosis. The degree to which oral cortisone helps in healing the cutaneous manifestations is difficult to assess. If lesions are few and disfiguring, local injections of hydrocortisone can be tried, but results are still uncertain. Calciferol in heavy dosage was given following its success in lupus vulgaris, but toxic sequelae were frequent. As some cases of sarcoidosis have hypercalcaemia which may produce renal failure, calciferol would not seem a suitable drug in the treatment of sarcoidosis. Happily, one can look forward to spontaneous resolution in many cases.

\section{REFERENCES}

PAL'TRIER, L M (r940), Maladie de Besnier-Boeck-Schaumann, Masson et Cie Paris.

WIGLEY, J. E. M., and MUSSO, L. A. (1951), Brit. F. Derm., 63, 398 .

LEVER, W. F. (1954), 'Histopathology of the Skin,' 2nd edition, J. B. Lippincott \& Co., Philadelphia, London, Montreal, p. 188 RLBIN, L., et al. (1956), F.A.M.A., r62, 953.

SAL NIDERS, T.S. (I 957), A.M.A.Arch. Derm., 76, 6 Io.

SHELLEY, W. B. (1957, in press), 'Transactions of the St. John's Hosp. Derm. Soc. 